\title{
Index
}

7/7 94, 203

9/11 145, 203

Airsprung 21, 63, 128, 134

Amesbury 19, 22, 97, 122, 171n.86

anti-discrimination policies 59, 75-9, 95-6, 98, 103-4

anti-racism 56-7, 72, 100-2, 104, 201

Arabic culture and language 22, 63, 65, $71,106,156-7,161$

Arabs 21-2, 26n.21, 65, 97, 131, 135, $140,162,168$ n. 38

assimilationist policies (education) 34 , 38-9, 46, 201

shift away from 67, 69-70, 74

Avon Rubber 128

Bath 19, 126, 130, 154-5

Birmingham 2, 4, 23, 30, 32-3, 37, 40, 42, 46, 50n.38, 57, 59, 64, 66, 75, $100,124-7,135,138-9,142-3,146$, 148, 157-60, 174n.160, 182-5, 187, 189, 192, 202

Bowyers 18, 21, 63, 128-9, 134, 152

see also working with pork

Bradford 2, 23, 33, 57, 64-5, 68, 89n.85, $124,182,185,187,189$

Bradford-on-Avon 21-2, 122, 125, 137, 139,162

Bristol 19, 50n.38, 64, 66, 96, 126, 129-30, 142, 146, 154-6, 159, $174 \mathrm{n} .160,184$

burials or burial rituals $64-5,67,102$, 107-9, 149-50, 152, 159-60, 162-3, 177n.222, 182, 184, 186, 188, 190-1 see also cemeteries; graves

buses, working on the $41,125,183$

businesses and businesspeople 41, 43, 63, 77-8, 122, 125-7, 129-31, 133-8, 163, 167n.26, 171n.86, 171n.91, $184,189,191$

see also entrepreneurialism and entrepreneurship; restaurants and takeaways; self-employment

Calne 18-19, 21-2, 61, 104, 122, 126, 131, 134, 137-8, 155, 191

Cameron, David 94

cemeteries 64, 107, 149, 184, 186, 191

see also burials or burial rituals; graves

census 10, 16n.53, 19-23, 26n.21, 27n.29, 31, 49n.11, 49n.12, 58, 82n.14, 82n.15, 82n.16, 83n.19, 91n.109, 96-7, 107, 200

Chippenham 19-22, 42, 60-1, 63, 71, $97,122,129,132-3,135,141,147$, 155,164

Commission for Racial Equality (CRE) 4, 13n.19, 59-61, 63, 78-9, 85n.36, 94, 186

Commonwealth Immigrants Act (1962) 29

Commonwealth Immigrants Act (1968) 29

community centres $24,45,104-5,140-2$, $150,154-5,163,187,190-1$

see also mosques

community or social cohesion $1,8-10$, 19, 24, 78, 94-6, 98, 103-5, 108, $127,131,142-4,151,162-4,179$, 184, 188-92, 201, 203 
community relations 32 , 43-6,

$$
\text { 59-63, 83n.22 }
$$

Community Relations Councils (CRCs)

9, 23, 43, 45-6, 59-60, 84n.24, 182

Corsham 20, 63, 74, 129-30

council housing 40-2, 63, 78-9, 181

see also housing

CRCs see Community Relations

Councils

CRE see Commission for Racial Equality curry houses and Indian and South

Asian food 126, 129, 132, 134,

163,188

see also restaurants and takeaways

Devizes 19, 21-2, 31, 42, 46, 71, 105, $107-8,122,127,134-5,137$, $141,144,155-6,161,163-4$, $171 \mathrm{n} .86,191-2$

discrimination 1-4, 8, 23, 29-30, 37, 44, $47,57,59-60,62-3,67-8,72-3$, 75-6, 78-9, 82n.12, 98, 101-2, 113n.55, 124, 127, 131, 145-6, 150, 162, 180, 186, 188, 191, 202, 204 see also harassment; Islamophobia; prejudice; racism; victimisation dispersal policy (education) 37-8

Eastern European migrants 5, 97, 186 education and schools 1-2, 8-9, 19, 22, 24, 30, 43, 53n.63, 57, 65, 80, 87n.70, 88n.81, 89n.85, 90n.100, 94-5, 112n.39, 201-4

experiences of in Wiltshire 130, 132, 145-7, 149, 159, 164

policies and measures in Wiltshire 32-9, 42, 44-6, 50n.38, 59-60, 62-3, 67-75, 78, 81, 98-100, 102, 104, 106, 109, 182-5

see also religious education

Eid 45, 123, 144, 149, 154, 191

employment $1,8,10,24,40-2,44,62$, 75-7, 81, 91n.109, 100, 124, 129-36, 146, 152, 162-4, 169n.47, 188

factory work in Wiltshire 21, 23-4, 44, 127-9, 131, 134, 152, 163, 169n.40, 189 see also Airsprung; Avon Rubber; Bowyers

English-language classes and acquisition 33-8, 42, 45, 69-70, 73-5, 99

entrepreneurialism and entrepreneurship $2,8,10,30,43,77-8,126-7,130-1$, 133-6, 162-3, 167n.26, 169n.49, $188,202-4$

see also businesses and businesspeople; restaurants and takeaways; self-employment equal opportunities policies 10, 30, 57, 59, 62-3, 72, 74-81, 90n.107, 95, $100-5,201$

\section{Gloucester 159}

graves 64, 107, 184

see also burials or burial rituals; cemeteries

halal food and butchers 57, 65, 102, 150, 152, 159-60, 162-3, 177n.218, $187-8,190$

halal or ritual slaughter 24, 64-7, 184 see also slaughterhouses; slaughterman licenses

harassment 3, 24, 62, 74, 79, 105, 121, $139,145-6,183$

see also discrimination; Islamophobia; prejudice; racism; victimisation

headscarf 146-8, 150, 187, 190

Highworth 102, 117n.110

Highworth Rural District Council 41

Highworth Town Council 184

home or property ownership 43, 136-7, 163,191

see also housing; mortgages

housing 2, 8-10, 30, 37, 39-42, 44, 53n.63, 57, 60, 62-3, 78-9, 81, 95, 102-3, 105, 124, 127, 131, 136-40, 164, 171n.97, 181-3, 185, 188, 201-4

see also council housing; home or property ownership; mortgages; neighbourhoods; residential concentration or segregation; residential dispersal 
Immigration Act (1971) 29, 44, 54n.75 Islamophobia 2, 8, 123, 143, 146, $188,203-4$

see also discrimination; harassment; prejudice; racism; victimisation

Italians in Wiltshire 20, 31, 33, 48n.9, 134

Jamaicans in Wiltshire 31, 134-6

kebabs, kebab shops and Turkish food 129-30, 132-3, 135, 163, 188

see also restaurants and takeaways

Kennet District Council 60, 77, 83n.22

Kenyan Asian Crisis 29, 32

Kenyan Asians 32, 51n.44

Koran or Quran 64, 157-8, 161

Leicester 2, 30, 39-40, 42, 46, 53n.63, $57-8,66,96,121,124,146$, 167n.18, 189

Local Government Act (1966) and Section 11 funding 70-1, 74, 88n.81

London or Greater London 2, 19, 21, 23, 32-3, 37, 39-40, 42, 58, 64, 68, 81n.1, 89n.85, 95, 100, 106, 116n.97, 121, 124-7, 129-30, 134-5, 138-9, 155-9, 164, 165n.6, 169n.41, 184-5, 187, 189, 192, 197n.53, 197n.54, 200-2

Brent 42, 58, 182-3

Ealing 37, 42, 183

Hackney 33, 79, 92n.120

Islington 33

Lambeth 182

Merton 100

Newham 23

Southall 37

Tower Hamlets 23, 58, 95, 124

Macpherson Inquiry and Report 94-103, 108, 112n.39, 185

Manchester and Greater Manchester 2, $57,68,81 n .1,124,126,182-3$

Marlborough 19, 21, 61, 71

MCA see Moroccan Community Association of Southern England and Wales
Melksham 19, 21-2, 42, 63, 71, 74, 104, $107-8,122,125-6,128-33,135$, 137-9, 141-2, 146-7, 155-6, 159, $163,184,189,191$

Melksham Town Council 107 migration to Wiltshire, causes of 124-31

Moroccan Community Association of Southern England and Wales (MCA) 105-6

Moroccan Islamic Association 64-6 mortgages 23, 40, 45

see also home or property ownership mosques 24, 63, 65-7, 107, 117n.110, 122-3, 130, 140-4, 149-59, 161, 163-4, 176n.185, 176n.186, 176n.189, 177n.202, 180, 186-91, 197n.53, 201

see also community centres; prayer spaces or rooms; storefront mosques

mother-tongue language classes and teaching 62, 70-1, 73-5, 140

multi-ethnic Muslim communities (in Wiltshire) 10, 131, 140-5, 151, 155-6, 163, 188-91

multi-occupation 41, 136

multicultural policies $8-10,56-7,59,62$, 67-76, 78, 80-1, 89n.85, 94-5, 99, $182,185,201$

neighbourhoods $10,38,65,95,103$, 131-2, 136-40, 162-4, 188-91, 201

Newcastle upon Tyne 30, 46, 57, 92n.120, 182-3

North and West Wiltshire Council for Racial Equality 61, 66

North Wiltshire Council for Racial Equality 61, 84n.24

North Wiltshire District Council 61, 83n.22, 102, 104

oral history 119-20 interviews in Wiltshire 10, 121-4 respondents' profiles 121-2 and the study of migration 17 n. $55,120-1$ 
Oujda 21, 97, 106, 127-9, 146

twinning with Trowbridge 97, 106-7, 116n.98, 116n.99, 116n.101, 146

peripatetic teachers and schools 33 , $37-8,88 \mathrm{n} .81$

Pewsey 42

Phillips, Trevor 94, 186

police 62 , 81n.1, 98, 100, 107, 125, 143-4

Polish in Wiltshire 20, 31, 33, 48n.9, 97, 134

Powell, Enoch 29

prayers and Friday prayers 45, 141, 148-9, 154-6, 161, 187, 189-91

see also prayer rituals; prayer spaces or rooms

prayer rituals 102, 148, 151, 190

see also prayers and Friday prayers; prayer spaces or rooms

prayer spaces or rooms 45, 65-6, 104, $107,109,122,140-1,148-9,151$, $153-5,162-3,174 \mathrm{n} .160,182$, $187,190-1$

see also mosques; prayers and Friday prayers; prayer rituals; Quaker Meeting House; storefront mosques

prejudice $3,8,10,24,65-6,69,73,100$, 107,113 n.55, 121, 131, 145-50, 162, 180, 186-7, 191, 202, 204 see also discrimination; harassment; Islamophobia; racism; victimisation

Quaker Meeting House 141, 155, 190 see also prayer spaces or rooms

Race Relations Act (1965) 29

Race Relations Act (1968) 29, 43

Race Relations Act (1976) 10, 23, 29, $32,47,56,58-61,63,67-8,73-80$, 81n.1, 185

Race Relations (Amendment) Act (2000) 95-103, 108, 112n.39, 185 racial equality policies or lack thereof 59-63, 79-80, 181-2, 184, 201 see also Wiltshire Racial Equality Council racism 2, 5, 8, 10, 30-1, 57, 67, 71-2, 78, $98,101-2,124,202-4$

institutional 94, 98, 100, 102, 113n.55, 163

rural 3-5, 7, 12n.15, 24, 96, 102, 109, $121,131,139,145-7,150,180-1$, 185-8, 196n.39, 200

see also discrimination; harassment; Islamophobia; prejudice; victimisation

Ramadan 45, 67, 108, 141, 149, 154, 161,187

Rampton Report 67

religious education 36, 50n.38, 57, 68-9, 72, 108, 130-1, 143, 152, 156-9, 162-3, 181, 188, 190

see also Standing Advisory Council on Religious Education

residential concentration or segregation

2, 21, 36, 38, 88n.81, 124, 136, $138-9,183$

residential dispersal vii-viii, 50n.36, 60, 73, 80, 136-41, 143-4, 153, 163-4, 181, 183-4, 186-91, 199, 204

restaurants and takeaways 124-6, 129-30, 133-6, 154, 163, 168n.27, $171 \mathrm{n} .86,188,191$

see also businesses and businesspeople; curry houses and Indian and South Asian food; entrepreneurialism and entrepreneurship; kebabs, kebab shops and Turkish food; self-employment

riots 56, 78, 81n.1, 87n.70, 94, 183, 203

Roundway Parish Council 107, 184

Royal Wootton Bassett or Wootton

Bassett 19-20, 22, 122, 125-6, 132, $143,145,154,159,173 n .134$

Rushdie Affair 8, 57, 66, 203

SACRE see Standing Advisory Council on Religious Education

Salisbury 18-19, 21-2, 39-42, 46, 52n.53, 60-1, 73-4, 78, 80, 84n.24, 97, 105, 107-8, 122, 125-6, 129, 132-4, 136-7, 141, 146, 149-50, 154-5, 157, 159, 161, 163-4, 171n.86, 182, 192 
Salisbury District Council 61, 83n.22, 102

Scarman Inquiry and Report 81n.1, 87n.70

self-employment 125,182

see also businesses and

businesspeople; entrepreneurialism and entrepreneurship; restaurants and takeaways

slaughterhouses 64, 159

see also halal or ritual slaughter; slaughterman licenses

slaughterman licenses 65, 191

see also halal or ritual slaughter; slaughterhouses

social services $9,61-2,76-7,103,183$

Southampton 42, 128, 159

Spanish in Wiltshire 134

Standing Advisory Council on Religious Education (SACRE) 74, 107

see also religious education

storefront mosques 153, 162, 176n.187, 187,190

see also mosques; prayer spaces or rooms

Swann Report 68, 71-2, 74, 87n.68, 89 n. 85,185

Swindon 18-23, 27n.29, 31-7, 39-47, 48n.9, 49n.11, 49n.13, 50n.36, 58, 60, 66-7, 69-71, 74, 78, 82n.15, 97, 102, 104-5, 117n.110, 122, 125, 129-34, 136-7, 139-41, 146, 154-5, 157, 159, 164, 181-4, 189, 191

Swindon and District Council for Community Relations 32, 43, 54n.70, 183

Swindon Council for Community Relations 43

Thamesdown and District Community Relations Council 23, 84n.24

Thamesdown and District Council for Racial Equality 23, 61-2
Thamesdown Borough Council 75, $77,83 n .22$

Tidworth 21, 97

Trowbridge 18-23, 26n.23, 35-7, 39, $42,46-7,60,63-7,71,74,78$, $80,97,104-7,122,127-32,134$, 136-9, 141-3, 146-7, 149, 154-6, 159-62, 169n.40, 175n.168, 184, 189, 191

twinning with Oujda 97, 106-7, 116n.98, 116n.99, 116n.101, 146

Trowbridge Town Council 106, 116n.98, 116n.99

Ugandan Asian Crisis 29-30, 32, 39, 187

Ugandan Asians 9, 32, 39-43, 46, 53n.63, 53n.65, 184, 187

underachievement (educational) 2, 67, $72,98-9,112 \mathrm{n} .40$

unemployment $2,41,75,77-8$

victimisation $8,121,147,186,188,203$ see also discrimination; harassment; Islamophobia; prejudice; racism

Vietnamese refugees 20, 59, 68

Warminster 19, 42, 107, 122, 126, 131-2, 154-5

West Wiltshire Council for Racial Equality 61, 84n.24

West Wiltshire District Council 63-5, 75-6, 78, 83n.22, 103, 181, 184

Wiltshire Racial Equality Council 22, 24, 62-3, 74, 84n.24, 98, 100, 102-3, $108,182-3,185$

see also racial equality policies or lack thereof

Wolverhampton 57, 124, 183

working with pork 152, 162-3, 190 see also Bowyers

Yugoslavians in Wiltshire 134 